13

\title{
Компенсация большого энергетического разброса ионов многозазорными сеточными отражателями времяпролетных масс-спектрометров: случаи катастроф $A_{4}, A_{5}, A_{6}, A_{7}$
}

\author{
() И.И. Пилюгин
}

Физико-технический институт им. А.Ф. Иоффре РАН, 194021 Санкт-Петербург, Россия

e-mail: i.pilyugin@mail.ioffe.ru

Поступило в Редакцию 27 ноября 2020 г.

В окончательной редакции 27 ноября 2020 г.

Принято к публикации 3 декабря 2020 г.

Рассмотрена задача компенсации начального энергетического разброса ионов многозазорными отражателями времяпролетных масс-спектрометров с сетками. Рассмотрены случаи катастроф $A_{4}, A_{5}, A_{6}, A_{7}$ для простейшего варианта распределения потенциалов. Показано, что многозазорные отражатели, функция времени пролета которых описывается катастрофами $A_{4}, A_{5}, A_{6}, A_{7}$, компенсируют гораздо бо́льшие энергетические разбросы ионов в сравнении с катастрофами $A_{2}$ и $A_{3}$. Полученные результаты можно использовать при конструировании бессеточных масс-спектрометров, компенсирующих большие начальные энергоразбросы ионов.

Ключевые слова: времяпролетный масс-спектрометр, энергоразброс ионов.

DOI: $10.21883 / J T F .2021 .05 .50703 .328-20$

\section{Введение}

В предыдущей работе [1] показано, что большие начальные разбросы ионов по энергиям во времяпролетном масс-спектрометре (ВПМС) с сетками могут быть скомпенсированы конструированием многозазорных отражателей. Там же [1] было показано, что функция времени пролета ионов может быть математически описана катастрофами $A_{n}[2,3]$ и рассмотрены случаи простейших катастроф $A_{2}$ и $A_{3}$.

В настоящей работе анализ будет продолжен для катастроф $A_{4}, A_{5}, A_{6}, A_{7}$. Достигаемый диапазон компенсации энергий ионов -

$$
k=U_{\max } / U_{\min },
$$

где $q U_{\max }$ и $q U_{\min }-$ максимальная и минимальная энергия ионов в зоне ионизации источника соответственно. Теоремы теории катастроф [2,3], утверждают, что при одном и том же диапазоне компенсации энергий ионов разрешающая способность ВПМС по фактору компенсации разброса ионов по энергиям $R_{\text {ref }}$ должна резко увеличиваться с ростом номера катастрофы $n$, где $R_{\text {ref }}=T_{\text {ref }} / 2 \Delta T_{\text {ref }}$, где $T_{\text {ref }}-$ среднее время пролета иона в приборе, а $\Delta T_{\text {ref }}-$ разброс времени пролета ионов в приборе по фактору компенсации энергоразброса.

Целью настоящей работы является рассчитать зависимость $R_{\text {ref }}=R_{\text {ref }}(n, k)$ для простейших конструктивных реализаций исследованных катастроф $(n=2-7)$ и простейшего пространственного распределения потенциалов для катастроф $A_{4}, A_{5}, A_{6}, A_{7}$.
Функция времени пролета, в дальнейшем (ФВП), многозазорого отражателя (рис. 1), согласно [1], равна

$$
\begin{aligned}
& T=\sqrt{\frac{m}{2 q}}\left(\frac{L}{\sqrt{U}}+\frac{4 d_{1}}{U_{1}}\left(\sqrt{U}-\sqrt{U-U_{1}}\right)+\frac{4 d_{2}}{U_{2}-U_{1}}\right. \\
& \times\left(\sqrt{U-U_{1}}-\sqrt{U-U_{2}}\right)+\ldots+\frac{4 d_{n-1}}{U_{n-1}-U_{n-2}} \\
& \left.\times\left(\sqrt{U-U_{n-2}}-\sqrt{U-U_{n-1}}\right)+\frac{4 d_{n}}{U_{n}-U_{n-1}} \sqrt{U-U_{n-1}}\right),
\end{aligned}
$$

где $m$ - масса иона, $L-$ суммарный дрейф ионов, $d_{n}-$ промежуток между $n$ и $n+1$ сетками, $U_{n}$ - потенциал $n+1$ сетки отражателя. Сетка 1 отражателя имеет

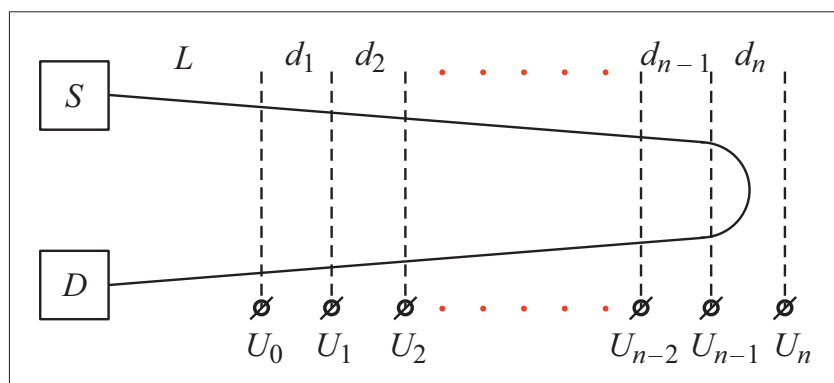

Рис. 1. Схема конструкции многозазорного сеточного отражателя ВПМС. $S-$ источник ионов, $D-$ детектор ионов, $L-$ дрейфовый промежуток движения ионов, $d_{1}, d_{2}, \ldots d_{n-1}, d_{n}-$ зазоры между сетками, $U_{0}, U_{1}, U_{2} \ldots U_{n-2}, U_{n-1}, U_{n}$ - потенциалы на соответствующих сетках. 
потенциал Земли $U_{0}=0$. Для дрейфового промежутка в случае, когда $U_{n-m-1}=U_{n-m}$, член в уравнении (2)

$$
\frac{4 d_{n-m}}{U_{n-m}-U_{n-m-1}}\left(\sqrt{U-U_{n-m-1}}-\sqrt{U-U_{n-m}}\right)
$$

заменяем на

$$
\frac{2 d_{n-m}}{\sqrt{U-U_{n-m}}}
$$

ФВП (2) может быть приведена к нормализованному виду, в котором безразмерная часть отделена от размерной:

$$
\begin{aligned}
T= & \sqrt{\frac{m}{2 q}} \frac{L}{\sqrt{U_{n-1}}}\left[\frac{1}{\sqrt{y}}+\frac{4 z_{1}}{v_{n}}\left(\sqrt{y}-\sqrt{y-v_{n}}\right)+\ldots\right. \\
& \ldots+\frac{4 z_{2}}{v_{n-1}-v_{n}}\left(\sqrt{y-v_{n-1}}-\sqrt{y-v_{n-2}}\right) \\
& \left.+\frac{4 z_{n-1}}{1-v_{3}}\left(\sqrt{y-v_{3}}-\sqrt{y-1}\right)+\frac{4 z_{n}}{v_{1}-1} \sqrt{y-1}\right] \\
= & \sqrt{\frac{m}{2 q}} \frac{L}{\sqrt{U_{n-1}}} \quad t\left(y ; z_{1} \ldots z_{n}, v_{1} \ldots v_{n}\right),
\end{aligned}
$$

где $y=U / U_{n-1}, \quad z_{n}=d_{n} / L$, а $v_{m+1}=U_{n-m} / U_{n-1}$ при этом $v_{2} \equiv 1$.

\section{1. Конструктивные особенности исследуемых отражателей и алгоритм расчета разрешающей способности}

Опишем распределения потенциалов исследуемых отражателей. Распределение потенциала в многозазорном отражателе является непрерывной функцией. Из работы [4] известно, что для получения фокусировки по энергии высокого порядка необходимо применить в отражателе ускоряющее поле. Поэтому рассмотрим следующую схему распределения потенциала (рис. 2, цифрами обозначены зазоры отражателя).

Из (2) и (5) легко получается ФВП для каждого случая рассматриваемых катастроф. Из условия фокусировки $n$ уравнений

$$
t_{y_{c p}}^{\prime}=0, \quad t_{y_{c p}}^{\prime \prime}=0, \quad \ldots, \quad t_{y_{c p}}^{(n)}=0,
$$

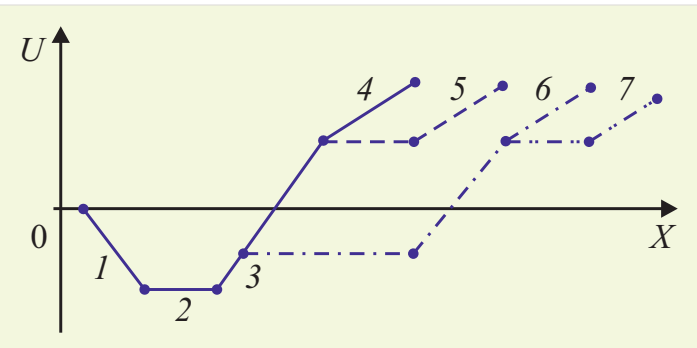

Рис. 2. Пространственное распределение потенциала отражателей.

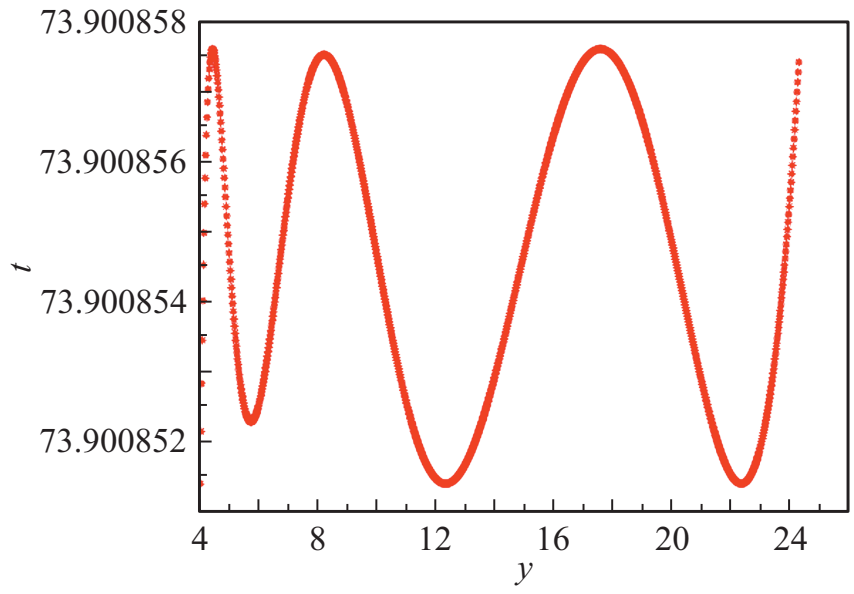

Рис. 3. Вид безразмерной функции времени пролета $t$ для максимума разрешающей способности многозазорного отражателя катастрофы $A_{6}$.

обращающихся одновременно в ноль в критической точке

$$
y_{c p}=\frac{U_{\max }+U_{\min }}{2 U_{n-1}}=\frac{v_{1}}{2}\left(1+\frac{1}{k}\right) .
$$

получается система $n$ линейных уравнений (6) относительно $z_{i}$ при случайным образом выбранных $v_{k}$. Система уравнений должна удовлетворять условию $z_{i}>0 \forall i$. Полученные $z_{i}$ вместе с $v_{k}$ являются первым приближением для многопараметрической оптимизации [5] разрешающей способности по $v$. В результате этой оптимизации получается набор чисел $v_{k_{1}}$, являющийся вместе с $z_{i}$ первым приближением многопараметрической оптимизации по $z$.

В результате проведения этой процедуры $N$ раз $(N>100)$ выбираем максимальное значение разрешающей способности $R_{\text {ref }}$ для заданного энергетического разброса $k$ (по формуле (1)) при определенных значениях параметров $z_{i}$ и $v_{k}$. Функция времени пролета для случая максимума разрешающей способности катастрофы $A_{6}$ изображена на рис. 3.

Результаты расчетов для всех исследуемых катастроф $A_{n}$ представлены на рис. 4. Дополнительно на рисунке приведены точки для катастроф $A_{2}$ и $A_{3}$ из работы [1].

Отметим, что для катастрофы $A_{7}$ данный алгоритм не срабатывает, и фокусировки седьмого порядка получить не удается. Данный алгоритм позволяет получить параметры отражателей с фокусировкой не более, чем шестого порядка по энергии.

\section{2. Обсуждение результатов и выводы}

Анализ рис. 4 показывает, что многозазорные сеточные отражатели ВПМС с числом зазоров 4-7 могут компенсировать начальные энергоразбросы ионов, редко встречающиеся на практике $(k>10)$. При этом достигаемая разрешающая способность $R_{\text {ref }}>10^{6}$, что 


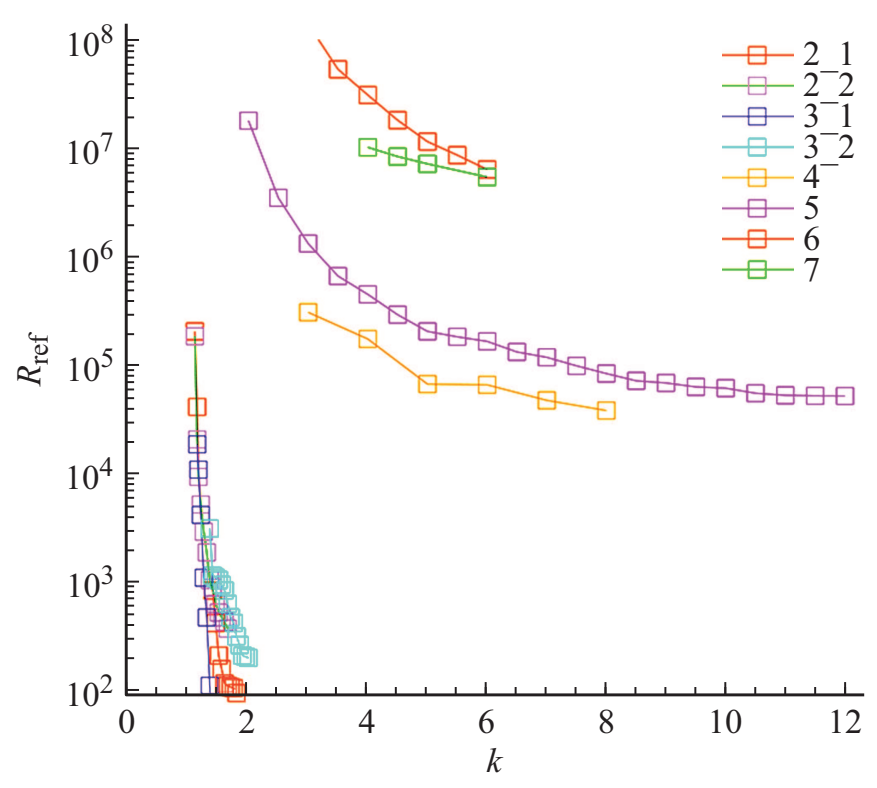

Pис. 4. Значения максимума разрешающей способности $R_{\text {ref }}$ многозазорного отражателя в зависимости от величины энергоразброса $k$. Номером у символа обозначена катастрофа $A_{n}$ : 2_1 - $A_{2}, 2 \_2-A_{2}, 3 \_1-A_{3}, 3 \_2-A_{3}, 4-A_{4}, 5-A_{5}$, $6-A_{6}, 7-A_{7}$.

практически позволяет полностью исключить фактор компенсации энергоразброса из аберраций, ограничивающих суммарную разрешающую способность ВПМС с сетками. Однако практически построить такой прибор невозможно, так как отношение $L / d_{2} \gg 50$ для всех исследованных в настоящей работе случаев, что не позволяет построить прибор разумных габаритов с большим значением отношения $L / d_{0}$, где $d_{0}$ - ширина пакета ионов в источнике. Чтобы построить такой прибор, необходимо сделать масс-спектрометр с большой величиной дрейфа $L$, т.е. сделать его многопроходным (два одинаковых отражателя расположены симметрично друг напротив друга и ионы входят в отражатели под небольшим углом), что технически невозможно с многосеточным отражателем, так как прозрачность сеток такого прибора делает его непригодным для использования на практике.

Единственной альтернативой представляется конструирование бессеточного отражателя, подобного рассмотреному в [4], с распределением потенциалов на „оси“ прибора, напоминающим рис. 2 для катастроф $A_{5}$ или $A_{6}$. (Катастрофа $A_{7}$ уже не дает выигрыша в разрешающей способности, что видно из рисунка.) Расчет такого прибора выходит за рамки данного исследования.

\section{Список литературы}

[1] И.И. Пилюгин. ЖТФ, 86 (3), 128 (2016).

[2] В.И. Арнольд, А.Н. Варченко, С.М. Гусейн-Заде. Особенности дифберенцируемых отображений. Издание второе, испр. (МЦ-НМО, М., 2004), 672 с.

[3] Р. Гилмор. Прикладная теория катастроф (Наука, М., 1981), т. $1,2$.

[4] А.Н. Веренчиков, М.И. Явор. Научное приборостроение. 14 (2), 38 (2004).

[5] Электронный ресурс. режим доступа: http://www.scilab.org.

\section{Конфликт интересов}

Автор заявляет, что у него нет конфликта интересов. 